\title{
BOTANY \\ Floristic diversity and vegetation analysis of the community forests of South-West Haryana, India
}

\author{
Harikesh, Himanshi, Somveer Jakhar* \\ Ecology Lab, Department of Botany, Kurukshetra University, Kurukshetra-136119, Haryana, India
}

Received: November 26, 2019

Revised: April 30, 2020

Accepted: May 08, 2020

Published: May 23, 2020

*Corresponding Author:

Somveer Jakhar,

Email: somveerjakhar@

hotmail.com

\begin{abstract}
Community forestry is an important form of forests and provides resources to over a half billion people in developing countries. They also play a significant part in mitigating the $\mathrm{CO}_{2}$ levels by sequestering a significant amount of carbon in the soil as well as biomass. The present paper assessed floristic diversity and vegetation structure in three different community forests of southwest Haryana which is a part of tropical dry deciduous forests. The vegetation sampling and data analysis were done following standard procedures. A total of 76 plant species belonging to 37 families in the form of 11 trees, 13 species of shrubs, 46 species of herbs, and 6 species of climbers are documented from all three sites. Poaceae was the most specious family in three sites. The highest tree diversity was recorded in Bhera forest followed by Daya and Dhanger. Regarding understory, the forest of Daya has a greater diversity than Bhera and Dhanger forests. Salvadora oleoides was the dominant tree species in Daya site and Dhanger site while in Bhera the dominant tree species was Ailanthus excelsa. The incidence of rampant livestock grazing and other anthropogenic disturbances were visible in all three sites which are primarily responsible for the degradation of these already fragmented village community forests.
\end{abstract}

KEYWORDS: Community forest, tropical dry deciduous forests, importance value index, biodiversity, phytosociology

\section{INTRODUCTION}

Haryana is a landlocked state in northwest India with a geographical area of $1.3 \%$ of the country. As the development is concerned, the state has well-developed agricultural as well as industrial sectors. However, the state has poor forest/tree cover compromising only about $3.62 \%$ of its total geographical area according to India State Forest Report, 2019. The area under forest and trees is an important indicator of its environmental condition. In the mid-1970s, worldwide research interest arose into the interrelation between people and trees which led to the establishment of the definitions of the term 'community forestry $[1,2]$. Learning the significance of community forests, virtually all countries around the world are working in the field of community forestry [3].

The importance of forests can be estimated by the fact that they provide half a billion with substantial livelihoods, from which millions of rural people living in poverty depend on community forests for their sustenance in this developing world. It is also found evident that community forests also provide various benefits along with plenty of livelihoods such as biodiversity conservation and carbon storage $[4,5]$. With contradiction to the forest products required by the ever-increasing population, the availability of forest resources is scarce. Inclusive of this, the non-sustainable means of resource extraction used by the people, due to lack of knowledge and ignorance among them causing the hazardous effects on the community forests. The species of an ecosystem are very important because their activities are essential processes that support and maintain those environments [6].

In Haryana, a small portion of land is occupied by community forests that are mainly present in the south-western region of the state. As, due to the presence of subtle soil moisture and water availability in the rest of the state, the area lately under community forests has now been converted into agricultural land. Hence, community forests are mainly confined to only a few districts in Haryana i.e., Hisar, Bhiwani, Fatehabad, Jhajjar, Sirsa, and Mahendergarh. Thus, the community forests of Haryana are very significant for study. Also, no study has been reported on these ecosystems by other workers from Hisar, Bhiwani, and Fatehabad.

Keeping this and the benefits as well as rapidly degrading conditions of community forests in mind, the present study revolves around the community forests of these three districts of Haryana. During this study, various parameters like Density, Basal area, IVI, and Diversity indices (Shannon-Weiner, Simpson, Pielou, and Margalef) were estimated to analyze the ecological conditions of the flora of selected community forests. 


\section{MATERIALS AND METHODS}

\section{Study Site}

The present investigation was carried out in community forest ecosystems of Daya village (Hisar), Dhangar village (Fatehabad), and Bhera village (Bhiwani) of south-west Haryana (Figure 1). These sites are located in the arid regions of Haryana and are influenced by the local steppe climate. There is a little rainfall during the year. The average temperature of Bhiwani is $25.2^{\circ} \mathrm{C}$ with annual precipitation of $465 \mathrm{~mm}$. The average temperature of Hisar is $25.1^{\circ} \mathrm{C}$ with about $459 \mathrm{~mm}$ of precipitation falls annually. While the average temperature in Fatehabad is $24.9^{\circ} \mathrm{C}$ with an annual rainfall of $390 \mathrm{~mm}$.

The selected community forests are natural ecosystems having significant biodiversity as well as a certain level of disturbance due to activities performed by local people like grazing of animals, tree felling for obtaining fuelwood, etc. The area occupied by the three community forests i.e., Daya village (Hisar), Dhangar village (Fatehabad), and Bhera village (Bhiwani) is 50 ha, 35 ha, and 22 ha respectively. Due to the lack of literature on the community forests of Haryana, the present study was carried out as an initiative in this direction.

\section{Vegetation Sampling}

For analyzing vegetation composition and plant diversity, the quadrat method was used in the selected community forests at the three sites, i.e., Daya village (Hisar), Bhera village (Bhiwani), and Dhanger village (Fatehabad), by sampling 10 quadrats of $20 \times 20 \mathrm{~m}$, randomly placed. The trees occurring within in each quadrat were quantized. The circumference of trees was measured at $1.37 \mathrm{~m}$ height above the ground. The shrubs and herbs were observed within sub-quadrats of size $5 \times 5 \mathrm{~m}$ and $1 \times 1 \mathrm{~m}$ respectively placed further within $20 \times 20 \mathrm{~m}$ quadrats.

\section{Analysis of Vegetation Composition}

The density (D), basal area (B.A.), and important value index (IVI) of the flora of selected sites were analyzed following Phillips, 1959 [7], and Mishra 1968 [8]. The importance value index (IVI) was calculated using Curtis and McIntosh, 1951 [9]. Along with this, disturbance status was also analyzed by calculating the Frequency class distribution pattern given by Raunkiaer, 1934 [10].

\section{Population Structure of Tree Species}

The population structure of trees was determined by girth class measurement and it was done according to NRSA Manual, 2008[11].

\section{Analysis of Species Diversity Indices}

The plant species diversity (H') was measured using the Shannon and Wiener equation [12] and concentration of dominance $(\mathrm{Cd})$ was determined by Simpson's index [13]. Equitability (E) was also calculated for the given vegetation using Pielou [14]. Whereas, Species richness (d) was calculated by Margalef's index [15].

\section{RESULTS}

\section{Vegetation Composition}

A total of 76 plant species including trees, shrubs, climbers and herbaceous plants were recorded in the community forest of the given three sites. The number of species was greater in the community forests of Daya, Hisar (54) as compared to that of the community forests of Bhera, Bhiwani (50), and Dhanger, Fatehabad (27).

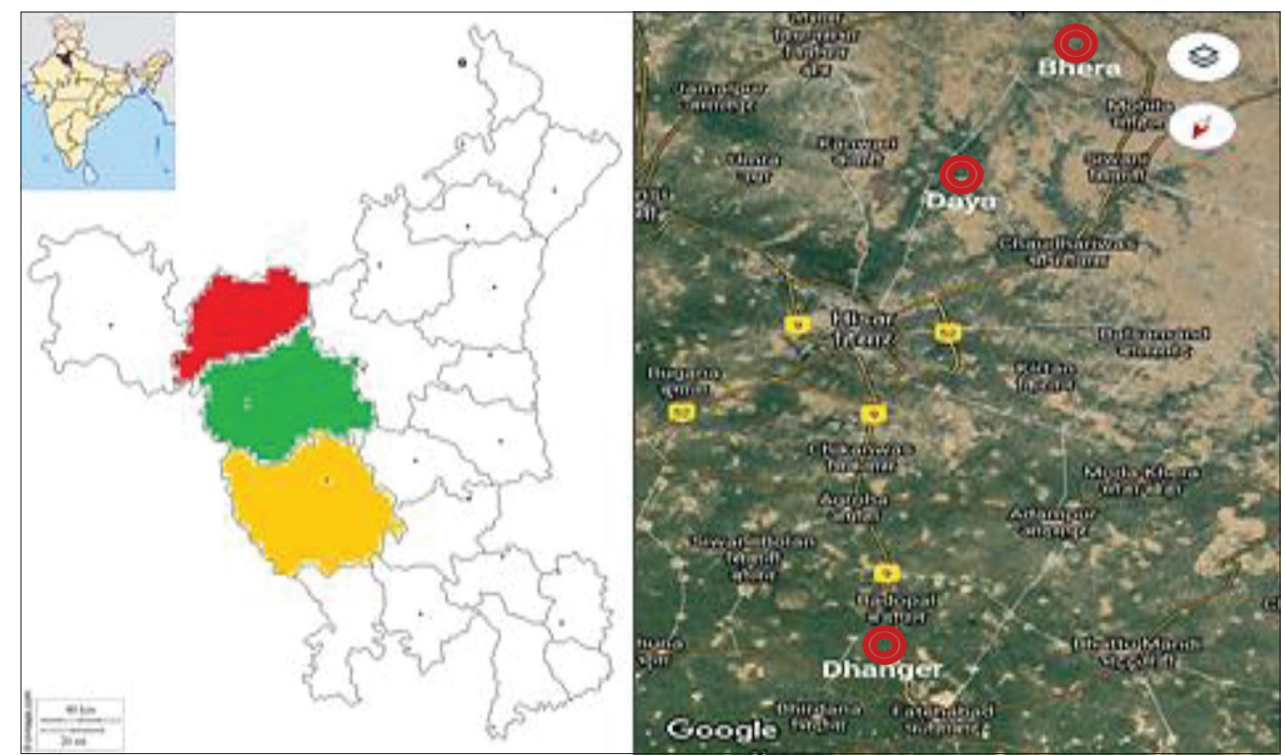

Figure 1: Location of study sites in Haryana, India (left), google imagery showing location of community forests in three districts with respect to each other (right) 
A total of 7 tree species were recorded in the community forest at Daya, which was dominated by Salvadora oleoides (IVI-71.47) followed by Prosopis juliflora (IVI 43.45), Acacia leucocephala (IVI 43.40) and Acacia nilotica (IVI 40.45). The IVI of other tree species varied from 24.88 to 39.36 . The IVI of various tree species in the community forest at Bhera varied from 5.75 to 87.69, dominated by Ailanthus excelsa (IVI 87.69), and followed by the co-dominance of Acacia nilotica (IVI 69.88) and Prosopis cineraria (IVI 49.79). The IVI of other tree species varied from 7.24 to 26.78 in this community forest. The community forest at Dhanger was dominated by Salvadora oleoides (IVI 117.57) followed by the co-dominance of Prosopis juliflora (IVI 113.93), Prosopis cineraria (IVI 34.51) and Acacia nilotica (33.97).

In shrub layer of dry deciduous forest at Bhera, the IVI value ranged from 2.14 to 126.64. Capparis decidua was the dominant shrub (IVI-126.64) followed by Parthenium hysterophorus (IVI85.55 ) and Calotropis procera (IVI-26.92). In the community forest at Bhera, Capparis decidua was the dominant shrub (IVI109.29) followed by Parthenium hysterophorus (IVI-98.59) and Lantana camara (IVI-33.92). The shrub layer of the community forest at Dhanger was dominated by Capparis decidua (IVI122.59) followed by Parthenium hysterophorus (IVI-105.09) and Abutilon indicum (IVI-57.40).

In the case of ground floor vegetation, a total of 33 herb species were found in the community forest at Daya village having IVI values ranging from 0.79 to 51.39 , dominated by Peristrophe bicalyculata (IVI-51.39). Whereas Dactyloctenium aegyptium was found to be dominant with an IVI value of 25.89 at Bhera among the total 29 species of herbs recorded from there. In the community forest of Dhanger village, 17 herbs were recorded of which Peristrophe bicalyculata was found to be dominant with IVI value of 143.31 .

In the case of climbers Tribulus terrestris (IVI-127.48) was dominant at Daya. Cucumis melo (IVI-165.13) was dominant at Bhera and at the community forest of Dhanger, Hedera helix (IVI-173.53) was found to be dominant.

The distribution pattern of plant species was also analyzed by dividing value of abundance by frequency and calculating the ratio of the two. The maximum number of plant species were found to show a contiguous pattern of plant distribution i.e., A/F ratio $>0.050$. Regular distribution was found completely absent while only three plant species were found to have random distribution i.e., Acacia nilotica and Prosopis cineraria in Bhera village community forest and Salvadora oleoides and Prosopis cineraria in Dhanger village community forest $(\mathrm{A} / \mathrm{F}$ ratio $=0.025$ to 0.050$)$.

\section{Species Diversity Indices}

The species diversity indices i.e., H', Cd, E, and d were also calculated for the flora of given community forests. The value of H' was found to be maximum for Bhera village community forest, followed by community forests of Daya village and Dhanger village respectively. Other than this, concentration of dominance i.e., Cd was observed maximum for Dhanger village community forest, followed by Bhera village community forest and Daya village community forest respectively. The value of $\mathrm{E}$ was found to be greatest for Dhanger village community forest, followed by Daya village community forest and Bhera village community forest respectively. While, the value of $\mathrm{d}$ was found to be maximum for Daya village community forest, followed by Bhera village community forest and Dhanger village community forest respectively.

\section{Frequency Class Distribution}

When frequency class distribution of different plant species in Daya village community forest was plotted on a graph it showed that maximum percentage of the total number of species is found in the frequency class $1-20 \%$ and the minimum number of species and minimum percentage of the total number of species are found in the frequency class $61-80 \%$ (Figure $2 \mathrm{a}$ ). In the case of Bhera village community forest, the maximum percentage of the total number of plant species is found in the frequency class $21-40 \%$ while the minimum number of plant species and minimum percentage of the total number of plant species are found in the frequency class $41-60 \%$ (Figure 2b). While at Dhanger village community forest, the maximum percentage of the total number of plant species was obtained by frequency class $1-20 \%$, and the minimum percentage of the number of plant species was shown by frequency class 41-60\% (Figure 2c).

A certain level of disturbance was recorded in all three community forests, as the frequency distribution curve was not found to be J-shaped as suggested by Raunkiaer (1934). A J-shaped curve of frequency distribution indicates that the ecosystem is intact with no signs of disturbance which was not deliberated for the three community forests indicating ecosystem disturbance in the given community forests.

\section{Population Structure}

The population structure of tree species in different community forests was also calculated (Figures 3-5) by assigning them to the girth classes as per to NRSA manual (2008). In the present study it was found that in the community forest of Daya village, the maximum number of tree species was found in the lowest girth class i.e., $0-30 \mathrm{~cm}$, such as Morus alba, Prosopis juliflora and Azadirachta indica. While in Bhera forest the maximum number of tree species was found to be in the girth class of $31-60 \mathrm{~cm}$ and $61-90 \mathrm{~cm}$ like Morus alba, Prosopis cinereria, and Pongamia pinnata. While in Dhanger forest Acacia nilotica, Prosopis cineraria, and Salvadora oleoides were found to belong to the girth class of 61-90 and 121-150.

\section{Pearson Correlation Analysis (PCA)}

Pearson correlation was also calculated for the three community forests (Table 1). It was found that density showed a positive correlation with the A/F ratio, Simpson index (Cd) and Margalef index (d) while it was found to be negatively correlated with the basal area (BA), Shannon Weiner index (H') and Pielou index 
(E). Other than this, BA was found to be positively correlated with $\mathrm{E}$ while negatively correlated with all other parameters i.e., D, A/F ratio, IVI, H', Cd, and d. H' was found to be positively correlated with $\mathrm{E}$ and negatively correlated with all the other parameters i.e., D, A/F ratio, IVI, H', Cd, and d.

\section{DISCUSSION}

Phytosociological studies are crucial for understanding the studies of any ecosystem. It also helps in understanding species interactions, ecosystem processes as well as services and change exerted by the external forces like various natural and anthropogenic disturbances. Many phytosociological studies have been accomplished in the forest ecosystems of Haryana [16-18]. In an ecosystem, plant species assemble in a particular manner and hence can help in vegetation mapping or quantification and evaluation. Thus, information regarding the floristic composition of natural ecosystems and habitat types becomes a key component for improving the long-term management of natural resources [19-21]. In the present study, it was found that the community forest of Daya site, Hisar is rich in plant diversity as revealed by its vegetation analysis in comparison to the other two sites i.e., Dhanger site in Fatehabad and Bhera site in Bhiwani district. The findings of the current study are equivalent to that of different ecosystems under tropical climates [22,23]. In the study, a total of 76 plant species belonging to 37 families were recorded from all three sites (Table 2-4). Similarly, Sahu et al., 2012 [24] recorded 57 species in dry deciduous forests of Eastern Ghats. Studies from the tropical dry deciduous forest in Sagar district reported a total of 36 trees, 8 shrubs, and 34 herbs [25]. Whilst a total number of 29 tree species belonging to 17 families were recorded across six sites from the tropical dry deciduous forests of Central India [26] and 14 tree species under 10 families were reported from Amarkutir, tropical dry deciduous forest of West Bengal [27].

The basal area of a tree is the circumference occupied at breast height (cbh) and is an important attribute to quantify the

Table 1: Pearson correlation for the different ecological parameters calculated during the study

\begin{tabular}{|c|c|c|c|c|c|c|c|c|}
\hline & D & A/F Ratio & BA & IVI & $\mathrm{H}$ & $\mathrm{Cd}$ & $E$ & $d$ \\
\hline D & 1 & $0.989 * *$ & -0.325 & 0.274 & -0.413 & 0.560 & -0.521 & $0.755 * *$ \\
\hline A/F Ratio & $0.989 * *$ & 1 & -0.378 & 0.276 & -0.455 & $0.615^{*}$ & -0.453 & $0.817 * *$ \\
\hline BA & -0.325 & -0.378 & 1 & -0.265 & -0.133 & -0.045 & 0.409 & -0.263 \\
\hline IVI & 0.274 & 0.276 & -0.265 & 1 & -0.158 & 0.276 & -0.083 & 0.307 \\
\hline $\mathrm{H}$ & -0.413 & -0.455 & -0.133 & -0.158 & 1 & $-0.961 * *$ & 0.065 & $-0.696 *$ \\
\hline $\mathrm{Cd}$ & 0.560 & $0.615^{*}$ & -0.045 & 0.276 & $-0.961 * *$ & 1 & -0.138 & $0.832 * *$ \\
\hline$E$ & -0.521 & -0.453 & 0.409 & -0.083 & 0.065 & -0.138 & 1 & -0.196 \\
\hline D & $0.755^{* *}$ & $0.817 * *$ & -0.263 & 0.307 & $-0.696 *$ & $0.832 * *$ & -0.196 & 1 \\
\hline
\end{tabular}

${ }^{* *}$ Correlation is significant at the 0.01 level (2-tailed). ${ }^{*}$ Correlation is significant at the 0.05 level (2-tailed)

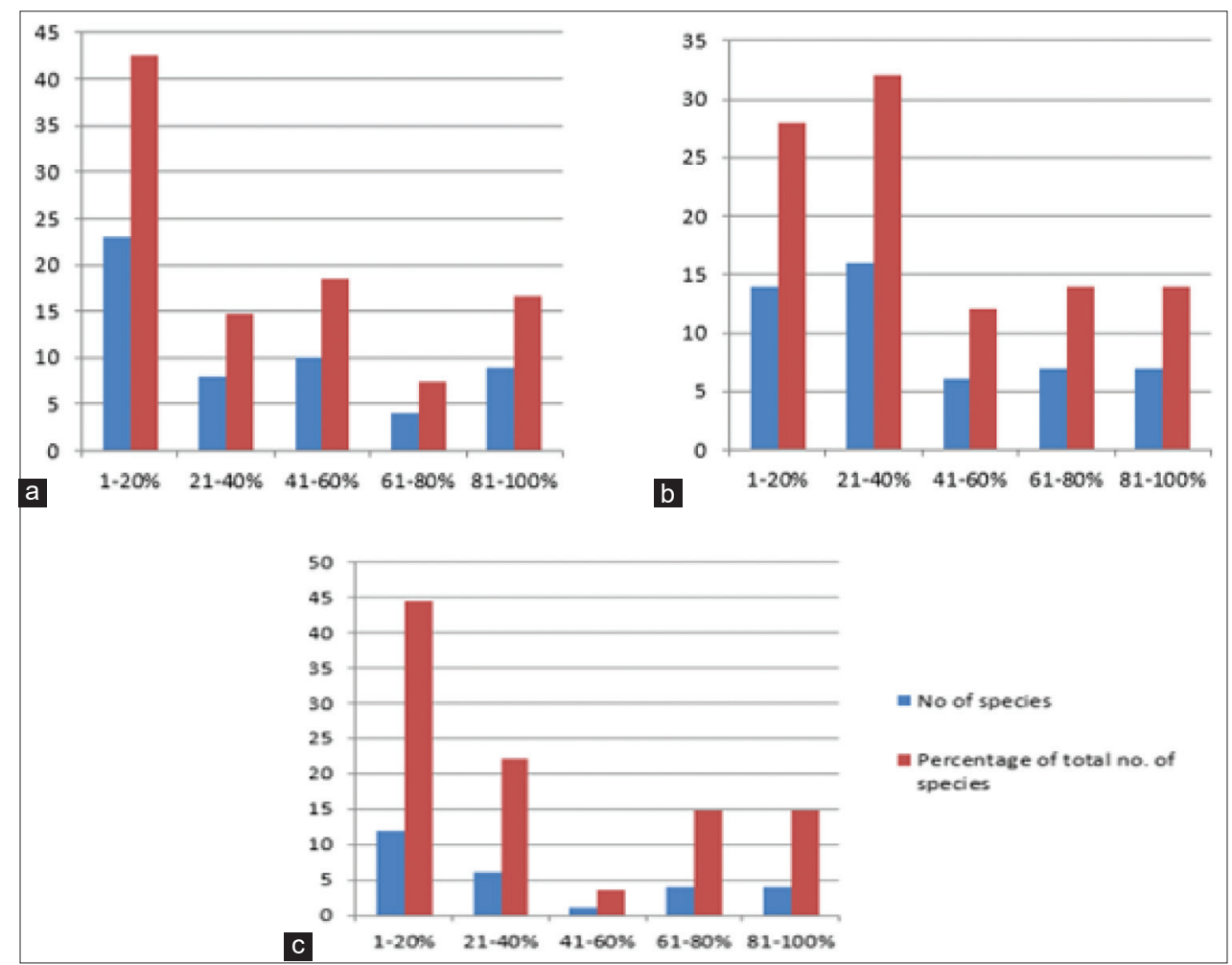

Figure 2: Frequency class distribution curve of the community forests of Daya, Hisar (a); Bhera, Bhiwani (b), and Dhanger, Fatehabad (c) 
vegetation structure and site quality [28]. In the present study, the basal area of the plant species varied significantly across the three sites Daya, Bhera and Dhanger village community forest. The basal area of trees in the three sites was found to be $23.739 \mathrm{~m}^{2} / \mathrm{ha}, 23.1048 \mathrm{~m}^{2} / \mathrm{ha}$, and $4.357 \mathrm{~m}^{2} /$ ha respectively, suggesting that the stand structure is quite healthy in all three sites. The values obtained are nearly in conformity with studies reported from some other workers $[27,29,30]$. The basal area of shrubs during the present study was calculated as $4.7449 \mathrm{~m}^{2} / \mathrm{ha}$, $0.3857 \mathrm{~m}^{2} / \mathrm{ha}$, and $0.5094 \mathrm{~m}^{2} / \mathrm{ha}$ for Daya, Bhera, and Dhanger

Table 2: Consolidated details of phytosociological analysis in the community forest ecosystem at Daya village, Hisar, Haryana, India

\begin{tabular}{|c|c|c|c|c|c|c|c|c|c|c|}
\hline S.N. & Name of the plant species & Family & $\begin{array}{c}\text { D } \\
\text { (ind./ha) }\end{array}$ & $\begin{array}{c}\text { A/F } \\
\text { Ratio }\end{array}$ & $\begin{array}{c}\text { B.A. } \\
\left(\mathrm{m}^{2} / \mathrm{ha}\right)\end{array}$ & IVI & $\mathrm{H}^{\prime}$ & $\mathrm{Cd}$ & $E$ & $d$ \\
\hline A & Trees & & & & & & & & & \\
\hline 1 & Acacia leucocephala & Mimosaceae & 80 & 0.128 & 1.24491 & 43.4019 & 0.27969 & 0.02093 & 0.14373 & 1.36922 \\
\hline 2 & Acacia nilotica & Mimosaceae & 52.5 & 0.058 & 1.95961 & 40.4504 & 0.27016 & 0.01818 & 0.13883 & 1.51484 \\
\hline 3 & Azadirachta indica & Meliaceae & 37.5 & 0.06 & 0.00178 & 24.8841 & 0.20650 & 0.00688 & 0.10612 & 1.65547 \\
\hline 4 & Dalbergia sissoo & Fabaceae & 15 & 0.15 & 6.98255 & 39.3641 & 0.26648 & 0.01721 & 0.13694 & 2.21561 \\
\hline 5 & Prosopis cineraria & Mimosaceae & 32.5 & 0.026 & 1.9929 & 36.9722 & 0.25801 & 0.01518 & 0.13259 & 1.72352 \\
\hline 6 & Prosopis juliflora & Fabaceae & 70 & 0.043 & 0.12456 & 43.4523 & 0.27985 & 0.02097 & 0.14381 & 1.41226 \\
\hline \multirow[t]{2}{*}{7} & Salvadora oleoides & Salvadoraceae & 32.5 & 0.052 & 11.43297 & 71.4747 & 0.34175 & 0.05676 & 0.17562 & 1.72352 \\
\hline & Total & & 320 & & 23.73928 & 300 & 1.90247 & 0.15613 & 0.97767 & 11.6144 \\
\hline B & Shrubs & & & & & & & & & \\
\hline 1 & Abutilon indicum & Malvaceae & 30 & 0.3 & 0.01735 & 5.7376 & 0.07567 & 0.00036 & 0.03286 & 2.64612 \\
\hline 2 & Calotropis procera & Asclepiadaceae & 145 & 0.058 & 0.0132 & 26.9229 & 0.21635 & 0.00805 & 0.09396 & 1.80841 \\
\hline 3 & Capparis sepiaria & Capparaceae & 65 & 0.072 & 0.48141 & 25.1863 & 0.20799 & 0.00704 & 0.09033 & 2.15600 \\
\hline 4 & Capparis decidua & Capparaceae & 425 & 0.17 & 4.17357 & 126.645 & 0.36405 & 0.17821 & 0.15810 & 1.48709 \\
\hline 5 & Ipomoea carnea & Convolvulaceae & 100 & 0.25 & 0.00042 & 12.4731 & 0.13222 & 0.00172 & 0.05742 & 1.95432 \\
\hline 6 & Maytenus heterophylla & Celastraceae & 7.5 & 0.3 & 0.00043 & 2.37245 & 0.03827 & 0.00006 & 0.01662 & 4.46671 \\
\hline 7 & Parthenium hysterophorus & Asteraceae & 1502.5 & 0.601 & 0.02495 & 85.5576 & 0.3578 & 0.08133 & 0.15539 & 1.23036 \\
\hline 8 & Phyllanthus reticulatus & Phyllanthaceae & 17.5 & 0.175 & 0.00058 & 4.8465 & 0.06664 & 0.00026 & 0.02894 & 3.14443 \\
\hline 9 & Urena lobata & Malvaceae & 2.5 & 0.1 & 0.00001 & 2.1485 & 0.03537 & 0.00005 & 0.01536 & 9.82221 \\
\hline \multirow[t]{2}{*}{10} & Ziziphus nummularia & Rhamnaceae & 30 & 0.133 & 0.03303 & 8.1088 & 0.09759 & 0.00073 & 0.04238 & 2.64612 \\
\hline & Total & & 2325 & & 4.74495 & 300 & 1.592 & 0.27785 & 0.69139 & 31.3618 \\
\hline C & Herbs & & & & & & & & & \\
\hline 1 & Achyranthes aspera & Amaranthaceae & 5175 & 2.07 & 0.09012 & 47.9062 & 0.29295 & 0.0255 & 0.08378 & 3.74199 \\
\hline 2 & Aerva lanata & Amaranthaceae & 2330 & 5.825 & 0.00753 & 11.7365 & 0.12679 & 0.00153 & 0.03626 & 4.12710 \\
\hline 3 & Aerva tomentosa & Amaranthaceae & 40 & 0.177 & 0.00149 & 2.86268 & 0.04439 & 0.00009 & 0.01269 & 8.67472 \\
\hline 4 & Ageratum conyzoides & Asteraceae & 32.5 & 0.325 & 0.0004 & 1.75513 & 0.03007 & 0.00003 & 0.00860 & 9.19212 \\
\hline 5 & Boerhavia diffusa & Nyctaginaceae & 5 & 0.2 & 0.000009 & 0.79168 & 0.01566 & 0.000006 & 0.00448 & 19.8827 \\
\hline 6 & Cenchrus ciliaris & Poaceae & 1205 & 3.012 & 0.00337 & 7.41708 & 0.09147 & 0.00061 & 0.02616 & 4.51070 \\
\hline 7 & Commelina benghalensis & Commelinaceae & 7.5 & 0.3 & 0.00009 & 0.82178 & 0.01616 & 0.000007 & 0.00462 & 15.8816 \\
\hline 8 & Crotalaria medicaginea & Fabaceae & 80 & 0.8 & 0.00011 & 1.80444 & 0.03075 & 0.000036 & 0.00879 & 7.30255 \\
\hline 9 & Croton bonplandianus & Euphorbiaceae & 5507.5 & 2.203 & 0.09268 & 49.5631 & 0.29746 & 0.02729 & 0.08507 & 3.71494 \\
\hline 10 & Cyamopsis tetragonaloba & Fabaceae & 7.5 & 0.3 & 0.00023 & 0.86179 & 0.01681 & 0.000008 & 0.00480 & 15.8816 \\
\hline 11 & Cynodon dactylon & Poaceae & 3937.5 & 1.575 & 0.00714 & 20.7496 & 0.18475 & 0.004784 & 0.05284 & 3.86552 \\
\hline 12 & Cyperus rotundus & Cyperaceae & 917.5 & 9.175 & 0.00779 & 6.3297 & 0.08141 & 0.000445 & 0.02328 & 4.69094 \\
\hline 13 & Dactyloctenium aegyptium & Poaceae & 3280 & 1.312 & 0.0105 & 19.88 & 0.17985 & 0.004391 & 0.05143 & 3.95276 \\
\hline 14 & Digera muricata & Amaranthaceae & 380 & 0.422 & 0.00642 & 7.5432 & 0.09260 & 0.000632 & 0.02648 & 5.38705 \\
\hline 15 & Digitaria ciliaris & Poaceae & 95 & 3.8 & 0.00007 & 1.0595 & 0.01994 & 0.000012 & 0.00570 & 7.02698 \\
\hline 16 & Eragrostis Spp & Poaceae & 3625 & 1.45 & 0.00087 & 18.088 & 0.16933 & 0.003635 & 0.04843 & 3.90453 \\
\hline 17 & Euphorbia hirta & Euphorbiaceae & 60 & 0.6 & 0.0007 & 1.9173 & 0.03229 & 0.00004 & 0.00923 & 7.81565 \\
\hline 18 & Euphorbia prostrata & Euphorbiaceae & 372.5 & 0.413 & 0.00011 & 5.7191 & 0.07549 & 0.000363 & 0.02159 & 5.40518 \\
\hline 19 & Evolvulus nummularius & Convolvulaceae & 95 & 0.422 & 0.00003 & 2.5985 & 0.04113 & 0.000075 & 0.01176 & 7.02698 \\
\hline 20 & Heliotropium strigosum & Boraginaceae & 5 & 0.2 & 0.000004 & 0.7902 & 0.01564 & 0.000006 & 0.00447 & 19.8827 \\
\hline 21 & Indigofera linnaei & Fabaceae & 25 & 0.1 & 0.00003 & 0.8533 & 0.01667 & 0.000008 & 0.00476 & 9.94135 \\
\hline 22 & Malvastrum coromandelianum & Malvaceae & 497.5 & 0.406 & 0.00236 & 7.4852 & 0.09208 & 0.000623 & 0.02633 & 5.15331 \\
\hline 23 & Oxalis corniculata & Oxalidaceae & 32.5 & 1.3 & 0.00002 & 0.8713 & 0.01696 & 0.000008 & 0.00485 & 9.19212 \\
\hline 24 & Paspalidium geminatum & Poaceae & 32.5 & 1.3 & 0.00004 & 0.8770 & 0.01705 & 0.000008 & 0.00487 & 9.19212 \\
\hline 25 & Peristrophe bicalyculata & Acanthaceae & 6955 & 4.346 & 0.09043 & 51.3978 & 0.30225 & 0.02935 & 0.08644 & 3.61695 \\
\hline 26 & Physalis minima & Solanaceae & 10 & 0.4 & 0.00115 & 1.1316 & 0.02104 & 0.000014 & 0.00602 & 13.8974 \\
\hline 27 & Pupalia lappacea & Amaranthaceae & 525 & 0.583 & 0.00549 & 7.6809 & 0.09383 & 0.000656 & 0.02683 & 5.10904 \\
\hline 28 & Setaria viridis & Poaceae & 12.5 & 0.5 & 0.000002 & 0.81055 & 0.01597 & 0.000007 & 0.00457 & 12.6696 \\
\hline 29 & Sida cordifolia & Malvaceae & 402.5 & 0.447 & 0.00611 & 7.5172 & 0.09237 & 0.000628 & 0.02642 & 5.33538 \\
\hline 30 & Solanum xanthocarpum & Solanaceae & 7.5 & 0.075 & 0.00003 & 1.5798 & 0.02762 & 0.000027 & 0.00790 & 15.8816 \\
\hline 31 & Trianthema portulacastrum & Aizoaceae & 130 & 5.2 & 0.00031 & 1.2255 & 0.02247 & 0.000016 & 0.00642 & 6.57417 \\
\hline 32 & Verbesina encelioides & Asteraceae & 5 & 0.2 & 0.00015 & 0.8319 & 0.01632 & 0.000007 & 0.00467 & 19.8827 \\
\hline \multirow[t]{2}{*}{33} & Xanthium strumarium & Asteraceae & 142.5 & 0.356 & 0.01415 & 7.5409 & 0.09258 & 0.000632 & 0.02648 & 6.45246 \\
\hline & Total & & 35935 & & 0.349935 & 300 & 2.68233 & 0.10149 & 0.76714 & 284.766 \\
\hline D & Climbers & & & & & & & & & \\
\hline 1 & Coccinia grandis & Cucurbitaceae & 90 & 0.225 & 0.00156 & 35.7788 & 0.25360 & 0.01422 & 0.18293 & 0.66669 \\
\hline 2 & Cucumis melo & Cucurbitaceae & 550 & 0.271 & 0.00581 & 124.532 & 0.36497 & 0.17231 & 0.26327 & 0.47544 \\
\hline 3 & Ipomoea pestigridis & Convolvulaceae & 57.5 & 2.3 & 0.00028 & 12.2020 & 0.13024 & 0.00165 & 0.09395 & 0.74041 \\
\hline \multirow[t]{2}{*}{4} & Tribulus terrestris & Zygophyllaceae & 265 & 0.424 & 0.02137 & 127.4871 & 0.36366 & 0.18058 & 0.26232 & 0.53766 \\
\hline & Total & & 962.5 & & 0.02902 & 300 & 1.11248 & 0.36878 & 0.80248 & 2.42021 \\
\hline
\end{tabular}


Table 3: Consolidated details of phytosociological analysis in the community forest ecosystem at Bhera village, Bhiwani, Haryana, India

\begin{tabular}{|c|c|c|c|c|c|c|c|c|c|c|}
\hline S.N. & Name of the plant & Family & $\begin{array}{c}\text { D } \\
\text { (ind./ha) }\end{array}$ & $\begin{array}{c}\text { A/F } \\
\text { Ratio }\end{array}$ & $\begin{array}{l}\text { B.A. } \\
\left(\mathrm{m}^{2} / \mathrm{ha}\right)\end{array}$ & IVI & $\mathrm{H}^{\prime}$ & $\mathrm{Cd}$ & $E$ & $d$ \\
\hline A & Trees & & & & & & & & & \\
\hline 1 & Acacia leucocephala & Mimosaceae & 27.5 & 0.068 & 0.22616 & 22.7435 & 0.19555 & 0.00574 & 0.08900 & 2.41386 \\
\hline 2 & Acacia nilotica & Mimosaceae & 100 & 0.048 & 1.63022 & 69.8899 & 0.3394 & 0.05427 & 0.15446 & 1.73717 \\
\hline 3 & Ailanthus excelsa & Simaroubaceae & 45 & 0.112 & 13.76369 & 87.6988 & 0.35952 & 0.08545 & 0.16362 & 2.10157 \\
\hline 4 & Azadirachta indica & Meliaceae & 17.5 & 0.077 & 1.10364 & 19.9638 & 0.18033 & 0.00442 & 0.08207 & 2.79505 \\
\hline 5 & Dalbergia sissoo & Fabaceae & 20 & 0.05 & 1.78917 & 26.7811 & 0.21568 & 0.00796 & 0.09816 & 2.67046 \\
\hline 6 & Ficus benghalensis & Moraceae & 2.5 & 0.1 & 1.451 & 10.1303 & 0.11441 & 0.00114 & 0.05207 & 8.73085 \\
\hline 7 & Morus alba & Moraceae & 5 & 0.2 & 0.22993 & 5.75451 & 0.07584 & 0.00036 & 0.03451 & 4.97067 \\
\hline 8 & Pongamia pinnata & Fabaceae & 7.5 & 0.3 & 0.36329 & 7.24080 & 0.08988 & 0.00058 & 0.04090 & 3.97041 \\
\hline \multirow[t]{2}{*}{9} & Prosopis cineraria & Mimosaceae & 50 & 0.040 & 2.54777 & 49.7970 & 0.29809 & 0.02755 & 0.13566 & 2.04497 \\
\hline & Total & & 275 & & 23.10487 & 300 & 1.86872 & 0.18751 & 0.85049 & 31.43506 \\
\hline B & Shurbs & & & & & & & & & \\
\hline 1 & Calotropis procera & Asclepiadaceae & 110 & 0.122 & 0.0079 & 27.3606 & 0.2184 & 0.00831 & 0.10502 & 1.48920 \\
\hline 2 & Capparis decidua & Capparaceae & 47.5 & 0.029 & 0.31805 & 109.295 & 0.36786 & 0.13272 & 0.17690 & 1.81312 \\
\hline 3 & Ipomoea carnea & Convolvulaceae & 22.5 & 0.9 & 0.00106 & 4.78393 & 0.06599 & 0.00025 & 0.03173 & 2.24826 \\
\hline 4 & Lantana camara & Verbenaceae & 160 & 0.4 & 0.04247 & 33.9251 & 0.24648 & 0.01278 & 0.11853 & 1.37926 \\
\hline 5 & Maytenus emarginata & Celastraceae & 5 & 0.2 & 0.00006 & 3.30516 & 0.04966 & 0.00012 & 0.02388 & 4.34934 \\
\hline 6 & Parthenium hysterophorus & Asteraceae & 982.5 & 0.484 & 0.01412 & 98.5981 & 0.36571 & 0.10801 & 0.17587 & 1.01595 \\
\hline 7 & Triumfetta rhomboidea & Tiliaceae & 85 & 0.85 & 0.00054 & 11.9457 & 0.12835 & 0.00158 & 0.06172 & 1.57563 \\
\hline \multirow[t]{2}{*}{8} & Ziziphus nummularia & Rhamnaceae & 22.5 & 0.1 & 0.00152 & 10.7855 & 0.11956 & 0.00129 & 0.05749 & 2.24826 \\
\hline & Total & & 1435 & & 0.38572 & 299.999 & 1.56203 & 0.26510 & 0.75117 & 16.1190 \\
\hline C & Herbs & & & & & & & & & \\
\hline 1 & Achyranthes bidentata & Amaranthaceae & 1205 & 0.594 & 0.00389 & 21.1140 & 0.18677 & 0.00495 & 0.05546 & 3.94686 \\
\hline 2 & Achyranthes aspera & Amaranthaceae & 167.5 & 0.744 & 0.00132 & 4.95523 & 0.06777 & 0.00027 & 0.02012 & 5.4677 \\
\hline 3 & Aerva javanica & Amaranthaceae & 470 & 0.752 & 0.00161 & 9.40441 & 0.10854 & 0.00098 & 0.03223 & 4.55082 \\
\hline 4 & Boerhavia diffusa & Nyctaginaceae & 227.5 & 0.364 & 0.00188 & 7.48501 & 0.09208 & 0.00062 & 0.02734 & 5.15924 \\
\hline 5 & Brachiaria reptans & Poaceae & 117.5 & 1.175 & 0.00077 & 3.24965 & 0.04901 & 0.00011 & 0.01455 & 5.87440 \\
\hline 6 & Cannabis sativa & Cannabaceae & 105 & 1.05 & 0.01039 & 12.4319 & 0.13192 & 0.00171 & 0.03917 & 6.01638 \\
\hline 7 & Cleom & Cleomaceae & 10 & 0.4 & 0.00011 & 0.92082 & 0.01776 & 0.000009 & 0.00527 & 12.1602 \\
\hline 8 & inabenghalensis & Commelinaceae & 190 & 0.844 & 0.00095 & 4.80003 & 0.06616 & 0.00025 & 0.01964 & 5.33635 \\
\hline 9 & Croton bonplandianus & Euphorbiaceae & 580 & 0.472 & 0.01124 & 21.1470 & 0.18696 & 0.00496 & 0.05552 & 4.40042 \\
\hline 10 & Cynodon dactylon & Poaceae & 1397.5 & 0.559 & 0.00221 & 21.9462 & 0.19131 & 0.00535 & 0.05681 & 3.8661 \\
\hline 11 & Dactyloctenium aegyptium & Poaceae & 1500 & 0.6 & 0.00534 & 25.8919 & 0.21143 & 0.00744 & 0.06279 & 3.82868 \\
\hline 12 & Datura innoxia & Solanaceae & 150 & 0.375 & 0.01466 & 18.4114 & 0.17127 & 0.00376 & 0.05086 & 5.58811 \\
\hline 13 & Digera muricata & Amaranthaceae & 292.5 & 0.182 & 0.00174 & 10.1080 & 0.11423 & 0.00113 & 0.03392 & 4.93091 \\
\hline 14 & Eragrostis spp. & Poaceae & 1260 & 0.504 & 0.00028 & 18.8453 & 0.17384 & 0.00394 & 0.05162 & 3.92219 \\
\hline 15 & Euphorbia hirta & Euphorbiaceae & 270 & 0.22 & 0.00215 & 9.57727 & 0.10996 & 0.00101 & 0.03265 & 5.00140 \\
\hline 16 & Euphorbia prostrata & Euphorbiaceae & 445 & 1.112 & 0.00348 & 10.2617 & 0.11545 & 0.00117 & 0.03428 & 4.59161 \\
\hline 17 & Malvastrum coromandelianum & Malvaceae & 62.5 & 0.277 & 0.00017 & 2.90008 & 0.04484 & 0.00009 & 0.01331 & 6.77119 \\
\hline 18 & Pedalium murex & Pedaliaceae & 517.5 & 0.575 & 0.01409 & 22.6140 & 0.19487 & 0.00568 & 0.05787 & 4.48071 \\
\hline 19 & Phyllanthus niruri & Phyllanthaceae & 380 & 0.422 & 0.00516 & 12.7498 & 3422 & & 0.03986 & 4.71366 \\
\hline 20 & Physalis minima & Solanaceae & 10 & 0.4 & 0.00004 & 0.85319 & 0.01667 & 0.000008 & 0.00495 & 12.1602 \\
\hline 21 & Poa annua & Poaceae & 587.5 & 0.94 & 0.00011 & 9.01155 & 0.10529 & 0.000902 & 0.03126 & 4.39155 \\
\hline 22 & Polygonum aviculare & Polygonaceae & 220 & 2.2 & 0.00163 & 5.00212 & 0.06826 & 0.000278 & 0.02027 & 5.19131 \\
\hline 23 & Polygonum persicaria & Polygonaceae & 145 & 5.8 & 0.00082 & 2.82057 & 0.04387 & 0.000088 & 0.01303 & 5.62618 \\
\hline 24 & Portulaca oleracea & Portulacaceae & 82.5 & 0.206 & 0.00051 & 4.13304 & 0.05903 & 0.00019 & 0.01753 & 6.34518 \\
\hline 25 & Pupalia lapacea & Amaranthaceae & 152.5 & 0.67 & 0.00202 & 5.49669 & 0.07328 & 0.000336 & 0.02176 & 5.56974 \\
\hline 26 & Sida cordifolia & Malvaceae & 57.5 & 0.143 & 0.00084 & 4.22711 & 0.06005 & 0.000199 & 0.01783 & 6.91053 \\
\hline 27 & Solanum xanthocarpum & Solanaceae & 30 & 0.075 & 0.00031 & 3.46779 & 0.05155 & 0.000134 & 0.01531 & 8.23239 \\
\hline 28 & Verbesina encelioides & Asteraceae & 470 & 0.378 & 0.01413 & 22.9503 & 0.04870 & 0.005852 & 0.01446 & 4.55082 \\
\hline \multirow[t]{2}{*}{29} & Xanthium strumarium & Asteraceae & 20 & 0.2 & 0.00165 & 3.22329 & 0.04870 & 0.000115 & 0.01446 & 9.34663 \\
\hline & Total & & 11122.5 & & 0.1035 & 300 & 2.94393 & 0.053422 & 0.87427 & 168.9316 \\
\hline D & Climbers & & & & & & & & & \\
\hline 1 & Cucumis melo & Cucurbitaceae & 632.5 & 0.3 & 0.00397 & 165.135 & 0.32862 & 0.30299 & 0.23705 & 0.62018 \\
\hline 2 & Ipomoea pestigridis & Convolvulaceae & 32.5 & 1.3 & 0.00015 & 10.0488 & 0.11376 & 0.00112 & 0.08206 & 1.14901 \\
\hline 3 & Tinospora cordifolia & Menispermacea & 67.5 & 0.168 & 0.00046 & 32.7672 & 0.24186 & 0.01193 & 0.17446 & 0.94963 \\
\hline \multirow[t]{2}{*}{4} & Tribulus terrestris & Zygophyllaceae & 557.5 & 0.454 & 0.00084 & 92.0485 & 0.36250 & 0.09414 & 0.26149 & 0.63256 \\
\hline & Total & & 1290 & & 0.00542 & 300 & 1.04676 & 0.41019 & 0.75507 & 3.3514 \\
\hline
\end{tabular}

village community forests respectively. This can be related to the range of basal area calculated for shrubs during a study of tropical dry deciduous forests of Haryana [18], i.e., $0.77 \mathrm{~m}^{2} / \mathrm{ha}$ to $2.87 \mathrm{~m}^{2} / \mathrm{ha}$. The total value of Basal area for shrubs, herbs and climbers was recorded as $1.48 \mathrm{~m}^{2} /$ ha during a study reported from tropical dry deciduous forests of Bundelkhand, Uttarpradesh [31]. Whilst, during the present study it was found to be $5.1177 \mathrm{~m}^{2} / \mathrm{ha}, 0.4946 \mathrm{~m}^{2} / \mathrm{ha}$ and $0.8052 \mathrm{~m}^{2} / \mathrm{ha}$ in Daya, 
Table 4: Consolidated details of phytosociological analysis in the community forest ecosystem at Dhanger village, Fatehabad, Haryana, India

\begin{tabular}{|c|c|c|c|c|c|c|c|c|c|c|}
\hline S.N. & Name of the plant & Family & D (ind./ha) & A/F Ratio & B.A. $\left(m^{2} / h a\right)$ & IVI & $\mathrm{H}^{\prime}$ & $\mathrm{Cd}$ & $\mathrm{E}$ & $d$ \\
\hline A & Trees & & & & & & & & & \\
\hline 1 & Acacia nilotica & Mimosaceae & 22.5 & 0.018 & 3.0181 & 33.9706 & 0.24665 & 0.01282 & 0.17792 & 0.96354 \\
\hline 2 & Prosopis cineraria & Mimosaceae & 30 & 0.033 & 4.013 & 34.5136 & 0.24877 & 0.01323 & 0.17945 & 0.88204 \\
\hline 3 & Prosopis juliflora & Fabaceae & 462.5 & 0.185 & 0.517 & 113.939 & 0.36768 & 0.14424 & 0.26523 & 0.48886 \\
\hline \multirow[t]{2}{*}{4} & Salvadora oleoides & Salvadoraceae & 60.25 & 0.045 & 32.809 & 117.576 & 0.36711 & 0.15360 & 0.26481 & 0.73197 \\
\hline & Total & & 575.25 & & 40.3571 & 299.999 & 1.23023 & 0.32390 & 0.88742 & 3.06642 \\
\hline B & Shrubs & & & & & & & & & \\
\hline 1 & Abutilon indicum & Malvaceae & 815 & 2.037 & 0.00195 & 57.4027 & 0.31642 & 0.03661 & 0.22824 & 0.44754 \\
\hline 2 & Calotropis procera & Asclepiadaceae & 12.5 & 0.125 & 0.00093 & 14.9053 & 0.14915 & 0.00246 & 0.10759 & 1.18777 \\
\hline 3 & Capparis decidua & Capparaceae & 57.5 & 0.143 & 0.46878 & 122.595 & 0.36569 & 0.16699 & 0.26379 & 0.74041 \\
\hline \multirow[t]{2}{*}{4} & $\begin{array}{l}\text { Parthenium } \\
\text { hysterophorus }\end{array}$ & Asteraceae & 1980 & 4.95 & 0.03777 & 105.096 & 0.36745 & 0.12272 & 0.26506 & 0.39521 \\
\hline & Total & & 2865 & & 0.50945 & 299.999 & 1.19872 & 0.32880 & 0.86469 & 2.77095 \\
\hline C & Herbs & & & & & & & & & \\
\hline 1 & Achyranthes bidentata & Amaranthaceae & 60 & 0.15 & 0.00046 & 6.7779 & 0.08563 & 0.00051 & 0.03022 & 3.90782 \\
\hline 2 & Alternanthera sessilis & Amaranthaceae & 222.5 & 2.225 & 0.0012 & 4.2588 & 0.0604 & 0.000202 & 0.02131 & 2.96026 \\
\hline 3 & Cenchrus ciliaris & Poaceae & 32.5 & 1.3 & 0.000009 & 1.7071 & 0.02941 & 0.000032 & 0.01038 & 4.59606 \\
\hline 4 & Croton bonplandianus & Euphorbiaceae & 5157.5 & 2.063 & 0.04017 & 44.193 & 0.28213 & 0.0217 & 0.09958 & 1.87173 \\
\hline 5 & Cynodon dactylon & Poaceae & 3165 & 2.583 & 0.00067 & 20.397 & 0.18278 & 0.00462 & 0.06451 & 1.98513 \\
\hline 6 & Cyperus rotundus & Cyperaceae & 237.5 & 9.5 & 0.0009 & 2.5845 & 0.04095 & 0.000074 & 0.01445 & 2.92495 \\
\hline 7 & $\begin{array}{l}\text { Dactyloctenium } \\
\text { aegyptium }\end{array}$ & Poaceae & 4642.5 & 2.292 & 0.00257 & 28.412 & 0.22322 & 0.00896 & 0.07878 & 1.89505 \\
\hline 8 & Eragrostis spp. & Poaceae & 5457.5 & 3.410 & 0.0009 & 28.522 & 0.22372 & 0.00903 & 0.07896 & 1.85943 \\
\hline 9 & Launaea nudicaulis & Asteraceae & 5 & 0.2 & 0.000112 & 1.6648 & 0.02882 & 0.00003 & 0.01017 & 9.94135 \\
\hline 10 & $\begin{array}{l}\text { Malvastrum } \\
\text { coromandelianum }\end{array}$ & Malvaceae & 47.5 & 1.9 & 0.000136 & 1.7922 & 0.03058 & 0.000035 & 0.01079 & 4.14429 \\
\hline 11 & Marsilea minuta & Marsileaceae & 150 & 6 & 0.000038 & 2.0466 & 0.03402 & 0.000046 & 0.01200 & 3.19320 \\
\hline 12 & Oxalis corniculata & Oxalidaceae & 62.5 & 2.5 & 0.000002 & 1.7889 & 0.03054 & 0.000035 & 0.01078 & 3.86925 \\
\hline 13 & $\begin{array}{l}\text { Peristrophe } \\
\text { bicalyculata }\end{array}$ & Acanthaceae & 16300 & 8.049 & 0.24547 & 143.31 & 0.3529 & 0.22822 & 0.12455 & 1.64966 \\
\hline 14 & Physalis minima & Solanaceae & 15 & 0.6 & 0.00011 & 1.6922 & 0.0292 & 0.000031 & 0.01030 & 5.90831 \\
\hline 15 & Sida cordifolia & Malvaceae & 37.5 & 0.166 & 0.00049 & 5.1097 & 0.06936 & 0.00029 & 0.02448 & 4.41459 \\
\hline 16 & $\begin{array}{l}\text { Trianthema } \\
\text { portulacastrum }\end{array}$ & Aizoaceae & 20 & 0.8 & 0.000099 & 1.7025 & 0.02934 & 0.000032 & 0.01035 & 5.34093 \\
\hline \multirow[t]{2}{*}{17} & Xanthium strumarium & Asteraceae & 27.5 & 0.275 & 0.00215 & 4.0305 & 0.05790 & 0.00018 & 0.02043 & 4.82773 \\
\hline & Total & & 35640 & & 0.29551 & 300 & 1.7909 & 0.27405 & 0.63212 & 65.2898 \\
\hline D & Climbers & & & & & & & & & \\
\hline 1 & Cucumis melo & Cucurbitaceae & 22.5 & 0.056 & 0.000063 & 126.464 & 0.36414 & 0.1777 & 0.52534 & 0.32118 \\
\hline \multirow[t]{2}{*}{2} & Hedera helix & Araliaceae & 80 & 3.2 & 0.000194 & 173.535 & 0.31664 & 0.3346 & 0.45682 & 0.22820 \\
\hline & Total & & 102.5 & & 0.000257 & 300 & 0.68078 & 0.5123 & 0.98216 & 0.54938 \\
\hline
\end{tabular}

Abbreviations: $\mathrm{D}=$ Density (individuals/hectare); $\mathrm{BA}=$ Basal Area $\left(\mathrm{m}^{2} /\right.$ hectare); $\mathrm{IVI}=$ Important value index; $\mathrm{H}^{\prime}=\mathrm{Shannon}$ Wiener Index; $\mathrm{Cd}=$

Simpson Index, $\mathrm{E}=$ Pielou Index, $\mathrm{d}=$ Margalef Index

Bhera and Dhanger site respectively. The high-value of B.A. in Daya site is due to the high density of Capparis decidua which was absent in the other two sites. The results suggest that the diversity and distribution of understorey species are scarce, thus require more attention and in need of management.

Tree density also varies from 15.0 to 462.5 in the present study (Tables 2-4). So much variation may imply that the land is not being fully utilized by the tree stand. The species diversity depends upon adaptations of species and increases with the stability of the community. The Shannon-Weiner (H') index of trees for all three sites varies from 1.23 to 1.902 which falls in the range of 0.67 to 4.05 reported in tropical forests of the Indian subcontinent [29-34]. On the other hand, the concentration of dominance (Simpson's index) in the present study is out of the reported range of $0.08-0.27$ in other forests $[26,30]$. In the present study, Cd for tree species varies from 0.156 to 0.323 for all three sites. However, Cd for herbs was found to be much lower while higher for shrubs and climbers, as compared to tree species in all three sites. This indicates the dominance of species in shrubs and climbers while diversity among trees and herbs. The value of Pielou index, E for trees was found to range between 0.85 to 0.977 and seen to follow the trend reported during the study of other workers [18,32]. The Margalef richness index for tree species occurred in the range of 0.32 to 23.72 for other tropical forests [26,29,33] for the two sites only i.e., Daya and Dhanger villages. Bhera site exhibited much higher values of 31.43 as compared to two other sites. With these values of diversity indices, it shows how the sites have lost tree species under the influence of man-made and ecological factors. In the present study, none of the sites showed the J-shaped curve as proposed by Raunkiaer (1934). The Inversed J-shaped curve is typical of well-developed forests with a broad range of tree sizes [35]. Invariably, none of the sites had the typical J-shaped curve which may reflect various degrees of disturbance in these sites. 


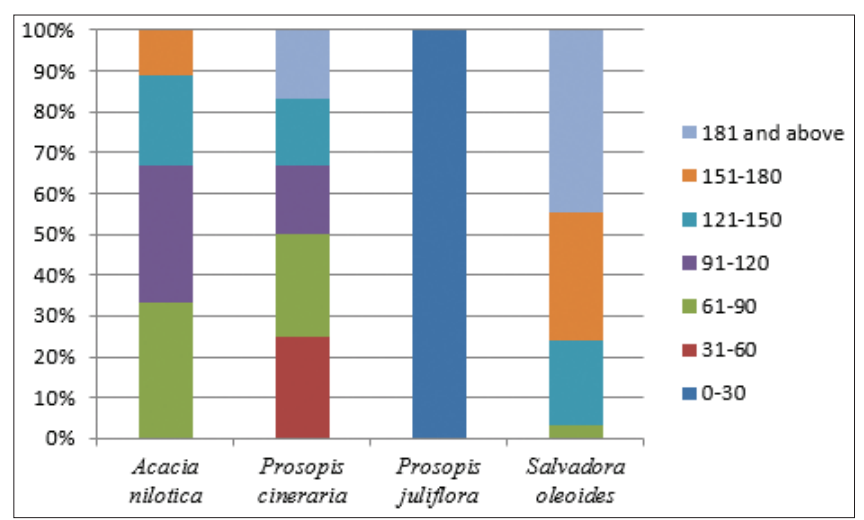

Figure 3: Graph representing the percentage of tree species of Dhanger village community forest belonging to the different girth classes

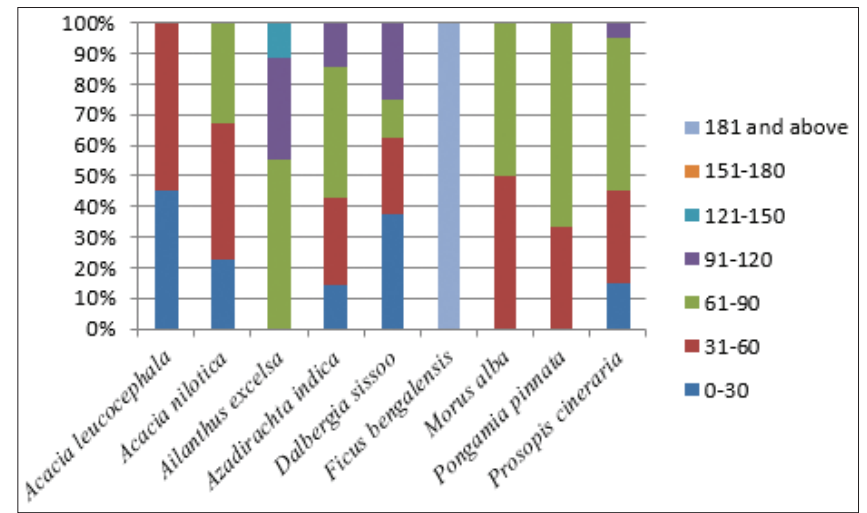

Figure 4: Graph representing the percentage of tree species of Bhera village community forest belonging to the different girth classes

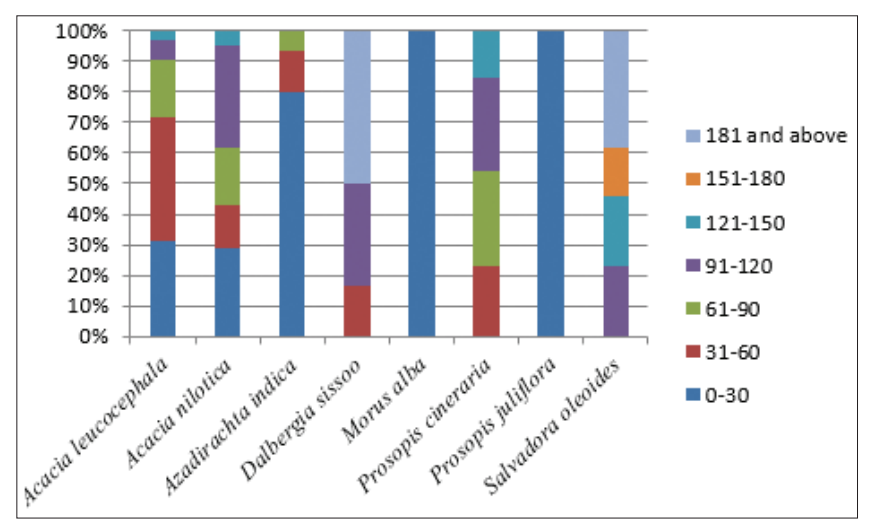

Figure 5: Graph representing the percentage of tree species of Daya village community forest belonging to the different girth classes

\section{CONCLUSIONS}

In the last two decades, the popularity of community forests has grown as reflected in the ratification of community forests related programs, policies, and laws. But, the community forests of Haryana are still lacking in gaining the attention of the state government. The present study sites were found to possess a significant amount of floristic composition and diversity in different strata. The Daya village community forest was found to be better flourished and diverse followed by Bhera and Dhanger village community forests respectively. But anthropogenic activities such as tree felling for fuelwood, grazing, encroachment, etc. create high pressure on these ecosystems leading to depletion in their state. Hence, steps should be taken in the field of community forest ecology to improve the fundamental quality of these ecosystems for their sustainability as well as their living flora and fauna. Some further studies are also warranted in this field to attain more knowledge regarding the community forestry in Haryana including level of disturbance and impact of grazing as well as spread of invasive plant species in them for the sustainability and management of these ecosystems.

\section{ACKNOWLEDGMENTS}

The authors acknowledge the Sarpanch of the three villages to allow the fieldwork in the selected community forests. Authors are also grateful to the Council of Scientific and Industrial Research, New Delhi for providing JRF to Harikesh and Himanshi. As well as, the authors also acknowledge Chairperson, Botany Department, Kurukshetra University, Kurukshetra for providing necessary laboratory facilities.

\section{REFERENCES}

1. Ford Foundation. Forestry for Sustainable Rural Development: A Review of Ford Foundation Supported Community Forestry Programs in Asia, Ford Foundation, New York. 1998.

2. Treue T. 'History of community forestry', unpublished lecture notes, The Royal Veterinary and Agricultural University, Denmark. 2004.

3. McCarthey J. 'Viewpoint - community forestry: a few sympathetic, but critical questions', Bulletin of the Global Caucus on Community Based Forest Management, Fall. 2004;11-12.

4. Chazdon RL. Beyond deforestation: restoring forests and ecosystem services on degraded lands. Science. 2008;320(5882):1458-1460.

5. Ranganathan J, Daniels R, Chandran S, Ehrlich PR, and Daily GC. Sustaining biodiversity in ancient tropical countryside. Proceedings of the National academy of Sciences of United States of America. 2008; 105(46): 17852-17854

6. Sanderson J. Landscape Ecology: A Top Down Approach. CRC press, Florida. 2020.

7. Phillips EA. Methods of Vegetation Study. Henry Holt and Co., Inc, New York. 1959.

8. Mishra R. Ecology Workbook. Oxford and IBH Publishing Co., New Delhi. 1968.

9. Curtis JT, Maclntosh RP. An upland forest continuum in the prairieforest border region of Wisconsin. Ecology. 1951;32:476-498.

10. Raunkiaer $\mathrm{C}$. The life forms of plants and statistical plant geography, ClarendonPress, Oxford. 1934; 632.

11. NRSA. Biodiversity Characterization at Landscape Level Using Remote Sensing and Geographic Information System. National Remote Sensing Agency, Department of Space, Govt. of India, Balanagar Hyderabad. 2008.

12. Shannon CE, Wienner W. The Mathematical Theory of Communication, University of Illinois, Urana, 1963.

13. Simpson EH. The measurement of diversity. Nature. 1949;163-688.

14. Pielou EC. The measurement of diversity in different types of biological collections. Journal of theoretical Biology. 1966;13:131-144.

15. Margalef R. Information theory in ecology. General Systematics. 1958;3:36-71.

16. Rout SK, Gupta SR. Analysis of forest vegetation of Morni Hills in north-east Haryana. Indian Academy of science (Plant Science). 1989;99(2):117-126.

17. Gupta SR, Kumar R. Vegetation composition and plant biodiversity in forest ecosystems of Siwaliks in northern Haryana. Indian Journal of Fundamental and Applied Life Sciences. 2014;4(2):76-88. 
18. Singh V, Gupta SR and Singh N. Vegetation composition, species diversity and soil carbon storage in tropical dry deciduous forests of southern Haryana. Indian Journal of Science. 2014; 7: 28-39.

19. Ewald J. A critique for phytosociology. Journal of Vegetation Science. 2003; 14: 291-296.

20. Kumar A, Bajpai O, Mishra AK, Sahu N, Behera SK, Bargali SS, Chaudhary LB. A checklist of the flowering plants of Katerniaghat Wildlife Sanctuary, Uttar Pradesh, India. Journal of Threatened Taxa. 2015;7(7):7309-3408.

21. Rout SD, Panda SK, Panda T. Phytosociological and floristic evaluation of Kuldiha Wildlife Sanctuary, Odisha, India. Tropical Plant Research. 2018;5(3):419-430.

22. Jaykumar R, Nair KKN. Beta diversity of angiosperms in the tropical forests of Nilgiri Biosphere Reserve, India. Tropical Ecology. 2012;53(2):125-164

23. Barua KN, Gogoi G, Hazarika P. Comparative study on structural composition and community association of Nambor Wildlife Sanctuary and its South-Westward extended Bornewria forest, Assam, India. Tropical Plant Research. 2018;5(2):233-242.

24. Sahu SC, Dhal NK, Mohanty RC. Tree species diversity, distribution and population structure in a tropical dry deciduous forest of Malygiri hill range, Eastern India. Tropical Ecology. 2012;53(2):163-168.

25. Thakur AS. Floristic composition, life-forms and biological spectrum of tropical dry deciduous forest in Sagar Districts, Madhya Pradesh, India. Tropical Plant Research. 2015;2(2):112-119.

26. Joshi RK, Dhyani S. Biomass, carbon density and diversity of tree species in tropical dry deciduous forests in Central India. Acta Ecologica Sinica. 2019;39(4): 289-299

27. Kumar ML, Nag A, Malakar S, Joshi HG. Population Structure and
Diversity of Trees in Amarkutir, A Tropical Dry Deciduous Forest of West Bengal, India. Indian Journal of Ecology. 2020; 47(1): 150-154.

28. Suthari S. Biodiversity characterization and aboveground vegetation carbon pool assessment in Northern Telangana at landscape leve using geospatial technique (PhD Thesis). Department of Botany, Kakatiya University, Warangal. 2013.

29. Kumar JIN, Kumar RN, Bhoi RK, Sajish PR. Tree species diversity and soil nutrient status in three sites of tropical dry deciduous forest of western India. Tropical Ecology. 2010; 51:273-279.

30. Sundarapandian SM, Swamy PS. Forest ecosystem structure and composition along an altitudinal gradient in the Western Ghats, South India. Journal of Tropical Forest Science. 2000;12:104-123.

31. Verma MK, Niranjan RK, Pal A. Phytosociological attributes of a tropical dry deciduous forest of Bundelkhand region of Uttar Pradesh, India. 2015; 3(10): 86-99.

32. Panda PC, Mahapatra AK, Acharya PK, Debata AK. Plant diversity in tropical deciduous forests of Eastern Ghats, India: A landscape level assessment. International Journal of Biodiversity Conservation. 2013;5:625-639.

33. Naidu MT, Premavani D, Suthri S, Venkaiah M. Assessment of tree diversity in tropical deciduous forests of Northcentral Eastern Ghats, India, Geology, Ecology, and Landscapes. 2018;2(3):216-227.

34. Lalfakawma SUK, Roy S, Vanlalhritpuia K, Vanlalhluna PC. Community composition and tree population structure in undisturbed and disturbed tropical semi-evergreen forest stands of North-East India. Applied Ecology and Environmental Research. 2009;7:303-318.

35. Mayer HA. Forest Mensuration. Pennsylvania Valley Publisher, State College, P.A. 1953;183 pp. 